\title{
LAS POSIBILIDADES DE LA TRADUCCIÓN PEDAGÓGICA EN LA ENSEÑANZA DEL ESPAÑOL COMO SEGUNDA LENGUA*
}

\author{
THE POSSIBILITIES OF PEDAGOGICAL TRANSLATION IN THE \\ TEACHING OF SPANISH AS A SECOND LANGUAGE
}

Laura Gasca Jiménez

University of Houston, Department of Hispanic Studies

RESUMEN

En las últimas décadas, la traducción ha ganado una creciente atención positiva como herramienta didáctica en la enseñanza de lenguas. Numerosos investigadores afirman que la traducción pedagógica tiene el potencial de facilitar el desarrollo de habilidades lingüísticas y conocimientos extralingüísticos (Carreres \& Noriega Sánchez, 2011; Cook, 2010; González Davies, 2004; Widdowson, 2003; Colina, 2002; Malmkjaer, 1998; Duff, 1989). El objetivo de este trabajo es investigar las posibilidades didácticas de la traducción en la enseñanza del español como segunda lengua. Primero, se ofrecerá una breve introducción teórica a la traducción pedagógica; segundo, se hará referencia a las prácticas pedagógicas de un curso avanzado de español y se presentará un análisis cualitativo y cuantitativo del impacto del uso de la traducción en dicho curso. Por último, se aportarán recomendaciones pedagógicas para integrar la traducción en la enseñanza del español como segunda lengua.

Palabras Clave: impacto pedagógico; habilidades lingüísticas; actitudes; traducción literal; técnicas de traducción

* Mi más sincero agradecimiento a la Dra. Alejandra Balestra por su apoyo incondicional a lo largo de este y otros muchos proyectos.

Estudios de Lingüística Aplicada, año 35, número 66, diciembre de 2017, pp. 205-232 doi: 10.22201/enallt.01852647p.2017.66.837 
Abstract

Over the past few decades, translation has gained renewed popularity as a pedagogical tool in language teaching. According to many researchers, translation has the potential to facilitate the development of linguistic skills and extralinguistic knowledge (Carreres \& Noriega Sánchez, 2011; Cook, 2010; González Davies, 2004; Widdowson, 2003; Colina, 2002; Malmkjaer, 1998; Duff, 1989). This study examines the didactic possibilities of translation into Spanish as an approach for teaching a second language. First, a brief theoretical introduction to pedagogic translation is outlined. Second, the pedagogical practices of an advanced Spanish course are described and quantitative and qualitative analyses of the impact of the use of translation are detailed. Finally, a series of pedagogical recommendations to incorporate the practice of translation into Spanish as a tool for teaching a second language are proposed.

KEYWORDS: pedagogical impact; linguistic abilities; attitudes; literal translation; translation techniques

Fecha de recepción del artículo: 25 de octubre de 2016

Fecha de recepción de la versión revisada: 17 de febrero de 2017

Fecha de aceptación: 28 de febrero de 2017

La correspondencia relacionada con este artículo debe dirigirse a:

Laura Gasca Jiménez

lgascajimenez@uh.edu

Estudios de Lingüística Aplicada, año 35, número 66, diciembre de 2017, pp. 205-232

doi: 10.22201/enallt.01852647p.2017.66.837 


\section{INTRODUCCIÓN}

En las últimas décadas, la traducción ha sido revisitada desde la enseñanza de segundas lenguas como herramienta didáctica. Esto debido a la creciente atención positiva que ha recibido por parte de expertos que destacan dicho valor didáctico (Carreres \& Noriega Sánchez, 2011; Cook, 2010; González Davies, 2004; Widdowson, 2003; Colina, 2002; Malmkjaer, 1998; Duff, 1989; entre otros), incluso después de la caída en desuso del método gramática-traducción.

El objetivo principal de este trabajo es demostrar las posibilidades didácticas de la traducción para el desarrollo de habilidades lingüísticas y conocimientos extralingüísticos en la enseñanza de segundas lenguas. Para ello, se hará referencia a las prácticas pedagógicas de un curso universitario de nivel avanzado de español y se ofrecerá un análisis cualitativo y cuantitativo del impacto del uso de la traducción a lo largo del curso. Por último, se aportarán recomendaciones pedagógicas para integrar la traducción en la enseñanza del español como segunda lengua.

\section{LA TRADUCCIÓN PEDAGÓGICA}

Por traducción pedagógica se entiende el uso de la traducción como herramienta didáctica para facilitar la enseñanza y la adquisición de lenguas. A pesar de los esfuerzos de muchos pedagogos por restablecer la traducción como herramienta didáctica, su uso sigue asociándose con el ya abandonado método gramática-traducción. En palabras de Carreres y Noriega Sánchez: "The word translation is equated in the minds of many language teachers with all the evils that accompanied the grammar-translation method, namely, a teacher-centered approach, a focus on grammar to the exclusion of other aspects of language, a disregard for oral and aural skills, decontextualization of language and student demotivation" (2011: 283). ${ }^{1}$

1 "La palabra traducción es equivalente, en la mente de muchos profesores, a todos los males que resultaron del método gramática-traducción: una metodología centrada en el profesor, un enfoque gramatical que excluye otros aspectos de la lengua, una desconsideración de las destrezas orales y auditivas, una descontextualización de la lengua y la desmotivación estudiantil" (traducción nuestra).

Estudios de Lingüística Aplicada, año 35, número 66, diciembre de 2017, pp. 205-232 doi: 10.22201/enallt.01852647p.2017.66.837 
Antes de la llegada del método directo, el método gramática-traducción había dominado el campo de la enseñanza de lenguas. El objetivo de este método era facilitar la lectura de literatura en la lengua meta, por lo que se trabajaban exclusivamente la comprensión lectora y la producción escrita. Las reglas gramaticales se analizaban y memorizaban para luego traducir oraciones desde y hacia la lengua meta. Con la llegada del método directo y el consecuente énfasis en la lengua hablada, la traducción fue expulsada de las clases de lengua para dar paso a lo que Cummins (2008) denomina el principio del monolingüismo, según el cual la primera y la segunda lengua se mantienen estrictamente separadas la una de la otra durante el aprendizaje.

Asociar la traducción con el método gramática-traducción ha dado lugar a cuatro críticas principales sobre esta práctica: en primer lugar, que es una actividad artificial que no tiene lugar dentro de una metodología comunicativa; en segundo lugar, que es un método restrictivo, ya que limita la práctica de la lengua exclusivamente a las destrezas de lectura y escritura; en tercer lugar, que puede ser una actividad contraproducente, dado que obliga al alumno a acceder a la segunda lengua a través de la lengua materna, lo que puede ocasionar interferencias, y, en cuarto lugar, que es un ejercicio frustrante y desalentador $(\mathrm{Ca}-$ rreres \& Noriega Sánchez, 2012).

Esta asociación ha sido ferozmente criticada desde la traductología y desde la enseñanza de lenguas y, en las últimas décadas, se han propuesto numerosos contraargumentos para reivindicar el ejercicio de la traducción con fines didácticos dentro de marcos de enseñanza comunicativos y de metodologías activas. A continuación, se ofrece una selección de los argumentos principales.

Carreres (2006) recurre al argumento, citado a menudo dentro del campo de la adquisición de segundas lenguas, de que la traducción es una estrategia que los estudiantes utilizan de forma natural para facilitar el proceso de adquisición de una segunda lengua. A este tipo de traducción se le denomina traducción interiorizada y consiste en establecer contrastes espontáneos con la lengua materna (Hurtado Albir, 2001: 644). Según Carreres (2006), incorporar la práctica de la traducción ayudaría a los estudiantes a racionalizar y sistematizar un mecanismo natural de aprendizaje.

Otros autores han destacado el potencial de la traducción para el desarrollo y el perfeccionamiento de habilidades y conocimientos lingüísticos. Duff (1989), 
uno de los primeros defensores de la traducción pedagógica, señala que esta práctica permite tomar conciencia de la influencia de la primera lengua en la segunda y lidiar con problemas de transferencia. Cook (2010) y González Davies (2002) enfatizan el desarrollo de la precisión gramatical, particularmente mediante la traducción inversa $(\mathrm{L} 1>$ L2). Según estos autores, la práctica de la traducción, a diferencia de la escritura libre, obliga a los estudiantes a enfrentarse a dificultades en su segunda lengua: "Let's think about what happens when students write a composition, for instance: They can avoid problematic areas and communicate effectively using avoidance strategies. With translation they have to relay what is in the source text, whether they are in command of the linguistic and extra-linguistic knowledge necessary to do so or not" (González Davies, 2002: 70). ${ }^{2}$

Cook (2010) destaca, además, el desarrollo de un conocimiento metalinguiístico mediante la reflexión y el debate alrededor de los problemas de traducción, ya que para poder justificar sus elecciones los estudiantes necesitan echar mano de un conocimiento declarativo sobre la lengua y sus características.

Zojer (2009) reitera muchos de los argumentos previos y añade que la traducción tiene un impacto muy positivo a niveles léxico y semántico. Según esta autora, la traducción contribuye a la expansión del conocimiento léxico del alumno, limita los errores de representación semántica y explica nuevo vocabulario. Incluso da un paso más allá al afirmar que, además de limitar la transferencia negativa, entendida como una desviación de las normas lingüísticas monolingües por influencia de otra lengua, contribuye al desarrollo de la competencia de transferencia, una subcompetencia de la competencia traductora que consiste en "saber comprender el texto original y reexpresarlo en la lengua de llegada según la finalidad de la traducción y las características del destinatario" (Hurtado Albir, 2001: 385).

Por su parte, Leonardi (2010) propone una serie de usos útiles de la traducción, principalmente: como herramienta crítica de lectura y como herramienta de análisis gramatical. De acuerdo con esta autora, al traducir se presta atención

2 "Pensemos en lo que ocurre cuando los estudiantes escriben una redacción, por ejemplo: pueden evitar áreas problemáticas y comunicarse efectivamente mediante el uso de estrategias de elusión. Con la traducción tienen que transmitir lo que se incluye en el texto de origen, tanto si tienen dominio del conocimiento lingüístico y extralingüístico necesario para hacerlo como si no" (traducción nuestra). 
a características lingüísticas y extralingüísticas que suelen ignorarse o analizarse superficialmente en actividades tradicionales de comprensión lectora. Además, afirma que la comparación de dos sistemas gramaticales mediante la traducción permite a los alumnos identificar las similitudes y las diferencias entre ambas lenguas para controlar la posible transferencia y, asimismo, mejora la conciencia y la precisión lingüísticas en su lengua materna.

González Davies agrega que la traducción permite desarrollar destrezas cognitivas como la habilidad de detectar y resolver problemas de traducción: "The students can look for titles of films and their translations or newspaper headlines in two or more languages and then discuss the puns or intertextual and cultural references that have been changed, or spot the mistake in mistranslations and try to give a more adequate translation, etc." (2002: 71). ${ }^{3}$ Además, según esta autora, dicha práctica favorece la adquisición de habilidades de documentación, ya que los estudiantes tienen que llevar a cabo búsquedas terminológicas y de otros tipos (textos paralelos, por ejemplo).

Sewell (2004), finalmente, cuestiona la creencia generalizada de que la traducción es una actividad frustrante para los estudiantes. Esta autora afirma que la naturaleza de la traducción escrita contribuye positivamente a la autoestima, ya que el traductor no es la fuente del mensaje original y no se ve involucrado en un acto inmediato de comunicación con el potencial de dañar su imagen personal. Además, sugiere que la traducción contribuye a la autonomía de los estudiantes, ya que permite la evaluación individual sin tener que depender exclusivamente del profesor.

En síntesis, los argumentos citados se oponen a las cuatro críticas mencionadas anteriormente y destacan que la traducción es una actividad motivante a la que los estudiantes recurren de forma natural y que su práctica permite trabajar diferentes destrezas, perfeccionar habilidades lingüísticas y extralingüísticas, así como controlar la transferencia negativa.

3 "Los estudiantes pueden buscar títulos de películas y sus traducciones o titulares de periódicos en dos o más lenguas y analizar los juegos de palabras, los cambios en las referencias culturales e intertextuales o identificar los errores en traducciones incorrectas y tratar de encontrar traducciones más acertadas, etc.” (traducción nuestra). 


\section{El CURSO}

Las prácticas pedagógicas y los materiales que se describen a continuación provienen de un curso avanzado de español dirigido a estudiantes de licenciatura ofrecido en la Universidad de Houston, Texas. Este curso forma parte de la especialización en español, a la que se accede opcionalmente tras cursar dos cursos básicos y dos cursos intermedios de español como segunda lengua. El objetivo general del curso es seguir desarrollando la competencia comunicativa de los estudiantes mediante la introducción de la práctica de la traducción directa (de español a inglés) e inversa (de inglés a español), con un particular énfasis en esta última modalidad. Los objetivos específicos incluyen desarrollar en los alumnos las habilidades de:

- Identificar las diferencias estructurales y culturales entre el inglés y el español.

- Resolver problemas básicos de traducción.

- Reconocer y emplear técnicas de traducción.

- Identificar los factores extralingüísticos que inciden en los textos al traducir.

Siguiendo las propuestas de Kiraly (2000) y González Davies (2017), este curso emplea una metodología activa, centrada en el estudiante, en la que se prioriza el proceso (la práctica de la traducción) sobre el producto (el texto traducido). Esta metodología permite que el alumno aprenda a traducir traduciendo, enfrentándose a los problemas que surgen en dicha práctica y adquiriendo estrategias de traducción para resolverlos.

Durante las primeras clases se introduce la traducción como una actividad comunicativa que depende de una serie de factores extralingüísticos, como la función del texto y la audiencia a la que se dirige. Además, se presentan diccionarios y otras fuentes de consulta que permiten que los estudiantes se enfrenten a la traducción como una actividad interpretativa y no como una operación matemática con una única respuesta correcta. A medida que avanza el curso, se introduce progresivamente terminología relacionada con la práctica profesional de la traducción; se explican las diferentes técnicas de traducción y su uso; se estudian y ponen en práctica los rasgos diferenciales principales entre el inglés y el español a niveles sintáctico, morfológico y de puntuación, y, por último, se ex- 
plora la dimensión social de la traducción y la importancia de considerar las variedades lingüísticas del español al traducir.

Antes de cada clase se les pide a los estudiantes que realicen una lectura teórica breve y que preparen una lista con tres conceptos clave abordados en la misma para facilitar el debate inicial de cada clase. Las lecturas provienen de dos manuales: Manual of Spanish-English translation (Washbourne, 2009) y Fundamentals of translation (Colina, 2015). El resto de la clase se dedica a la práctica de la traducción mediante actividades colaborativas. A continuación se incluye una selección de las actividades del curso: ${ }^{4}$

\section{Actividad 1: Paráfrasis}

Objetivos:

- Preparar a los estudiantes para la traducción de textos.

- Aprender a parafrasear y a cambiar de registro.

Materiales:

- Dos textos periodísticos breves en español.

Interacción:

- Primero en parejas y luego con toda la clase.

Procedimientos:

1. Los alumnos se organizan en parejas, toman turnos y se dictan mutuamente uno de los textos. A diferencia de los dictados tradicionales, los estudiantes tienen que escribir el mensaje del texto periodístico usando sus propias palabras, no las mismas que se les dictan.

2. La clase comenta las paráfrasis de todos los compañeros, haciendo énfasis en el registro de las palabras usadas.

Esta actividad se presta como calentamiento y es ideal para las primeras clases, ya que prepara a los alumnos para la traducción de textos sin tener que trabajar con las dos lenguas todavía. Para que efectúen un cambio de registro claro se recomienda usar textos periodísticos de registro formal.

4 Muchas de las actividades del curso fueron inspiradas por la obra de González Davies (2004). 
Actividad 2: Comparar traducciones

Objetivos:

- Identificar diferentes elecciones de traducción.

- Practicar la revisión en pareja.

Materiales:

- Dos traducciones de un mismo texto de origen.

Interacción:

- Primero en parejas y luego con toda la clase.

Procedimientos:

1. Los alumnos se organizan en parejas y reciben dos traducciones de un mismo texto de origen. Se les pide que identifiquen las diferencias entre los dos textos.

2. Ante toda la clase, presentan las diferencias que han encontrado y tratan de justificar las diferencias haciendo referencia a los factores extralingüísticos de los textos (objetivo, audiencia, tiempo, ${ }^{5}$ etc.).

Para poder aprovechar al máximo esta actividad se recomienda que se fomente la reflexión sobre la motivación de los cambios con preguntas como: ¿a qué se deben los cambios observados?, ¿se deben a un cambio de audiencia, por ejemplo?

\section{Actividad 3: Traducir en equipo}

Objetivos

- Fomentar la identificación de errores en la traducción y la edición de textos en la lengua meta.

- Practicar la toma de decisiones en grupo.

- Aprender a justificar elecciones tomadas colectivamente.

Materiales

- Un texto breve en inglés para traducir.

Interacción

- Primero en parejas, luego en grupos y con toda la clase.

Procedimientos

1. En parejas, los alumnos traducen un texto breve. Se les permite usar fuentes de consulta. Preparan una única traducción.

5 El parámetro tiempo se refiere al momento en el que el texto estará disponible al público. 
2. Se juntan con otra pareja, comparan las traducciones de los dos grupos, se ponen de acuerdo y preparan una versión final a partir de las dos versiones. Un miembro del equipo escribe la versión final en un documento de Google Docs. El resto de los grupos sigue el mismo procedimiento.

3. Se proyecta el documento de Google Docs ante toda la clase y se lleva a cabo un análisis contrastivo de las versiones finales.

\section{Actividad 4: Edición en equipo}

Objetivos

- Fomentar la identificación de errores en la traducción y la edición de textos en la lengua meta.

Materiales

- Textos breves de diferentes géneros traducidos mediante la herramienta Google Translate.

Interacción

- Primero en parejas y luego con toda la clase.

Procedimientos

1. Los alumnos se organizan en parejas, se les pide que comparen el texto de origen y la traducción producida por Google Translate, que identifiquen posibles problemas de traducción y que propongan alternativas para resolverlos.

2. Se discuten las propuestas ante toda la clase.

Además de las actividades colaborativas en clase, cada semana los estudiantes trabajan de forma individual en una traducción asignada. Todos reciben el mismo texto de origen, preparan un borrador y, el último día de clase de la semana, siguiendo un formato de taller, los estudiantes presentan sus traducciones, reflexionan con el resto de la clase sobre sus elecciones y toman notas para preparar una versión final. La versión final la entregan de forma individual junto con una breve reflexión sobre las características del texto, las principales dificultades a las que se enfrentaron durante la traducción y las soluciones que pusieron en práctica.

La primera traducción asignada es un fragmento de 185 palabras en inglés sobre la Universidad de Houston (véase Anexo C). Se trata de un texto es- 
tructuralmente sencillo pero que requiere de una investigación minuciosa para solucionar dificultades de traducción terminológicas, dadas las diferencias culturales que existen entre el inglés y el español en el campo de la educación universitaria.

En cuanto a los métodos de evaluación de estas actividades, se favorece una evaluación formativa. Para evaluar las traducciones asignadas, se usa el baremo de evaluación propuesto por González Davies (2003: 16-17). Este baremo fue diseñado para la evaluación de traducciones en un contexto de aprendizaje, no en uno profesional. Los estudiantes reciben una calificación individual por cada una de las traducciones y se les da la oportunidad de revisar y corregir las distintas versiones a partir de los comentarios y sugerencias de la profesora, y pueden volver a entregarlas.

Para el proyecto final los estudiantes llevan a cabo un ensayo de reflexión en el que responden a la siguiente pregunta: ¿cómo ha cambiado tu percepción de la traducción como producto, proceso y disciplina? Para responder a dicha pregunta se les pide que hagan uso de la terminología y de los conceptos teóricos introducidos a lo largo del curso.

\section{Metodología}

\subsection{Preguntas de investigación}

Las preguntas de investigación que guiaron el análisis de las posibilidades didácticas de la traducción fueron las siguientes:

1) ¿Cuáles son los hábitos de traducción e interpretación ${ }^{6}$ de los estudiantes avanzados de español como segunda lengua? ¿Y cuáles son sus actitudes al traducir?

2) ¿Qué impacto tiene la práctica de la traducción en el aprendizaje?

6 El término traducción suele reservarse para textos escritos y el de interpretación suele emplearse para la traducción de discursos orales. 


\subsection{Participantes}

Los participantes fueron 8 estudiantes de español matriculados en el curso descrito en la sección previa. Todos provenían de la Universidad de Houston y se encontraban en una estancia en el extranjero, en Cádiz, España, cuando tomaron el curso. La duración del curso fue de un mes con cuatro encuentros por semana (de lunes a jueves) de dos horas de duración cada uno. Dos estudiantes fueron descartados por no haber estado presentes durante la administración de todos los instrumentos. Así, el total de participantes se redujo a 6 (5 mujeres y 1 hombre).

\subsection{Procedimiento}

Los participantes completaron un cuestionario sobre sus hábitos de traducción y actitudes sobre dicho ejercicio y realizaron una actividad de traducción inversa previa (pre-test) y posterior (post-test) a dicho cuestionario. El test consta de cuatro preguntas cerradas de selección múltiple sobre la frecuencia con la que desempeñan tareas de interpretación y traducción en las combinaciones inglés-español y español-inglés; cuatro preguntas abiertas de respuesta corta sobre los contextos en los que tienen lugar dichas actividades y, finalmente, una pregunta abierta sobre sus actitudes ante la traducción en general (véase Anexo A). El texto de origen de la actividad de traducción es un fragmento de 160 palabras de una noticia publicada en inglés en la página web Yahoo News sobre el lanzamiento de un nuevo producto de Apple (véase Anexo B).

Para codificar el ejercicio de traducción se diseñaron dos baremos de evaluación a partir del trabajo de Valdés (2003). El primero de ellos permite evaluar la cantidad de información transferida. Para esto, el texto de origen se dividió en 13 cláusulas y se determinó el mensaje esencial de cada una. Cada cláusula del texto meta recibió una puntuación de 0 a 2, dependiendo de la cantidad de información esencial transmitida:

$2=$ Mensaje de partida transmitido totalmente (mensaje no deteriorado)

$1=$ Mensaje de partida transmitido parcialmente (mensaje deteriorado)

$0=$ Mensaje de partida no traducido o reproducido erróneamente 
El segundo baremo permite identificar las técnicas de traducción empleadas por los participantes para transmitir los mensajes del texto original. Siguiendo la clasificación de Hurtado Albir (2001), se diseñó una clasificación de las técnicas más empleadas en traducción (véase Anexo D). En cada cláusula del texto meta se identificaron las estrategias empleadas y, además, se codificó si estas se habían empleado satisfactoriamente (0-1). El análisis cuantitativo de las encuestas y de las traducciones se llevó a cabo mediante el programa Statistical Package for the Social Sciences (SPSS).

\section{RESUltAdOS Y DISCUSIÓN}

\subsection{Hábitos y actitudes}

El análisis cuantitativo de las encuestas indica que, antes de comenzar el curso, los estudiantes interpretaban con regularidad (21\% lo hacía cada día; $36 \%$, cada semana; $14 \%$, cada mes, y $29 \%$, raramente) y traducían ocasionalmente $(21 \%$, cada semana; $29 \%$, cada mes, y 50\%, raramente). El análisis cualitativo de las preguntas de respuesta abierta señala que los contextos más comunes de interpretación y traducción son el contexto personal, el laboral y el académico. A diferencia de la interpretación, la traducción parece llevarse a cabo casi exclusivamente como actividad de clase y como herramienta de aprendizaje personal de los estudiantes. Sus actitudes ante la traducción y la interpretación son muy positivas, y la mayoría hizo referencia al valor instrumental de la traducción como profesión y a su valor como herramienta didáctica: ${ }^{7}$

(1) Translation is extremely important because there is never an exact direct translationyou have to take into consideration context, audience, author, purpose, etc. Translation is one of the most useful and needed type of work a person could do because language is used to communicate by all people, and to continue growing and connecting

7 Las muestras de escritura se han reproducido tal y como las escribieron los estudiantes. La abreviatura $\mathrm{P}$ se refiere a participante. 
as a society and throughout the world, we need translation for that to be possible. Translation has been happening from the beginning of time. $(\mathrm{P} 3)^{8}$

(2) For me, translating passages is the way I can see that my vocabulary and understanding of the language has gotten better. Because I don't like to speak in Spanish, translating is what works best for me. (P5) ${ }^{9}$

En síntesis, al comenzar el curso, la mayoría de los estudiantes interpretaba con frecuencia y aproximadamente la mitad de ellos recurría a la traducción como herramienta de estudio. Además, sus actitudes eran muy positivas y destacaban el potencial didáctico de esta actividad. Estas tendencias apoyan, al menos parcialmente, los argumentos de Carreres (2006) sobre la traducción como mecanismo natural de aprendizaje y las opiniones de Sewell (2004) sobre el carácter alentador de la traducción.

\subsection{Impacto de la traducción en el proceso de aprendizaje de una L2/del español como L2}

La media de información esencial transferida para todos los participantes en la actividad de traducción fue de $53 \%$ en el pre-test y de $69 \%$ en el post-test (véase Cuadro 1).

Se llevó a cabo un $t$-test para muestras relacionadas con el objetivo de comparar la media de información transferida antes y después del curso y se encontró una diferencia significativa entre las dos $\left(t_{5}=-4.00, p=0.010\right)$, lo que sugiere que la práctica de la traducción tuvo un impacto positivo en el desarrollo de habilidades de transferencia en la traducción inversa. Este hallazgo apoya los ar-

8 "La traducción es sumamente importante porque nunca hay una traducción directa y exacta, tienes que tener en cuenta el contexto, la audiencia, el autor, el objetivo, etc. La profesión de traductor es una de las más útiles y necesarias que una persona podría tener porque todos usamos la lengua para comunicarnos, para seguir avanzando y conectarnos como sociedad en todo el mundo; necesitamos la traducción para que esto sea posible. La traducción ha estado presente desde el principio de los tiempos" (traducción nuestra).

9 "Traducir pasajes me permite ver cómo ha mejorado mi vocabulario y comprensión de la lengua. Como no me gusta hablar en español, traducir es lo que mejor me va" (traducción nuestra). 
gumentos de Zojer (2009) sobre el desarrollo de una competencia de transferencia mediante la práctica de la traducción.

CuADRo 1. Resultados estadísticos de la transmisión de información en la actividad de traducción

\begin{tabular}{lcccccccc}
\hline & N & Rango & Mín. & Máx. & Media & De & Asimetría & Curtosis \\
\hline Pre-test & 6 & 75 & 18 & 93 & 53 & 25 & 0.373 & 0.996 \\
Post-test & 6 & 57 & 36 & 93 & 69 & 23 & -0.381 & -1.164 \\
\hline
\end{tabular}

${ }^{*} t_{5}=-4.00, p=0.010$

Se codificó y analizó un total de 175 traducciones (90 en el pre-test y 85 en el post-test). Las técnicas de uso más frecuente tanto en el pre-test como en el post-test fueron la transposición (pre-test: 27\%; post-test: 32\%), la traducción literal (pre-test: $24 \%$; post-test: $21 \%$ ) y el préstamo (pre-test: $18 \%$; post-test: $17 \%$ ). La transposición es una técnica compleja que implica un cambio obligatorio o facultativo de categorías gramaticales (véase Anexo D). Su alta frecuencia de aparición sugiere que los participantes intentaron alejarse, no siempre de forma satisfactoria, de una traducción palabra por palabra tanto en el pre-test como en el post-test. Es importante tener en cuenta que la traducción literal es estructuralmente posible en aquellos contextos en los que el inglés y el español comparten estructuras paralelas, por lo que no debería concluirse, dada su alta frecuencia de aparición, que los participantes produjeron en su mayoría traducciones literales agramaticales. Cabe destacar también que el uso de préstamos es esperado en la traducción de aparatos informáticos, por lo que su alta frecuencia de uso no apunta necesariamente a dificultades léxicas.

La precisión media para todos los participantes en el uso de técnicas de traducción fue de $50 \%$ en el pre-test y de $66 \%$ en el post-test (véase Cuadro 2).

Se llevó a cabo una prueba $t$-test para muestras relacionadas con la finalidad de comparar la precisión media en el uso de técnicas de traducción antes y después del curso y se encontró una diferencia significativa entre las dos $\left(t_{5}=-3.287\right.$, $p=0.022)$. Este resultado sugiere que la traducción tuvo un efecto positivo en el perfeccionamiento del uso de técnicas de traducción. 
Cuadro 2. Precisión en el uso de técnicas de traducción

\begin{tabular}{lcccccccc}
\hline & N & Rango & Min. & Máx. & Media $^{*}$ & De & Asimetría & Curtosis \\
\hline Pre-test & 6 & 53 & 22 & 75 & 50 & 21 & 0.045 & 0.845 \\
Post-test & 6 & 42 & 50 & 92 & 66 & 16 & 0.943 & 0.845 \\
\hline
\end{tabular}

${ }^{*} t_{5}=-3.287, p=0.022$

$\mathrm{Al}$ analizar los niveles de precisión de las tres técnicas más frecuentes por separado, podemos observar que la precisión aumentó en las tres, particularmente en el caso de la transposición y del préstamo (véase Cuadro 3). El aumento de precisión en el uso de la transposición es particularmente revelador, ya que implica un mayor dominio de estructuras morfosintácticas en la segunda lengua. La mayor precisión observada en el uso de préstamos parece indicar un mayor control del léxico en la segunda lengua.

Cuadro 3. Precisión en el uso de transposiciones, traducciones literales y préstamos

\begin{tabular}{lccccc}
\hline & \multicolumn{3}{c}{ Pre-teSt } & & \multicolumn{2}{c}{ Post-TEST } \\
\cline { 2 - 3 } \cline { 5 - 6 } \cline { 5 - 6 } & N & $\%$ & & $N$ & $\%$ \\
\hline Transposición & $9 / 25$ & 36 & & $16 / 27$ & 59 \\
Traducción literal & $14 / 22$ & 64 & & $12 / 18$ & 67 \\
Préstamo & $10 / 16$ & 63 & & $12 / 14$ & 86 \\
\hline
\end{tabular}

En síntesis, el análisis cuantitativo señala que hubo una mejora significativa en la cantidad de información transferida al traducir de inglés a español, así como en la precisión en el uso de técnicas de traducción en español.

El análisis cualitativo de los proyectos llevados a cabo a lo largo del curso pone de manifiesto que, al finalizarlo, los estudiantes mostraron la capacidad de realizar traducciones inversas, identificar las diferencias estructurales entre el inglés y el español, reflexionar críticamente sobre los factores extralingüísticos que rodean los textos y un mayor control sobre las traducciones literales agramaticales.

En las primeras traducciones encontramos múltiples ejemplos en los cuales los estudiantes aplican las reglas morfosintácticas del inglés en el español, por ejemplo: el uso generalizado de la yuxtaposición y la coordinación; la tendencia a anteponer los adjetivos, independientemente de su clase; el uso de sustantivos 
con función adjetiva; la presencia categórica del sujeto; la tendencia a colocar el verbo al final de la oración, y la omisión del artículo definido. Los ejemplos de estas tendencias que mostramos en el Cuadro 4 provienen de la primera traducción, asignada en la primera semana del curso. Como se explicó anteriormente, esta tarea consistía en la traducción de un texto informativo sobre la Universidad de Houston. Por limitaciones de espacio se incluye solo un número limitado de ejemplos de estas tendencias. ${ }^{10}$

Cuadro 4. Ejemplos de tendencias en las traducciones presentadas

\begin{tabular}{|c|c|c|}
\hline TENDENCIAS & TEXTO DE ORIGEN & TEXTO META \\
\hline \multirow[t]{2}{*}{ Anteposición de adjetivo } & $\begin{array}{l}\text { At the graduate level, we offer } 139 \text { master's } \\
\text { [sic], } 54 \text { doctoral, and three professional } \\
\text { degree programs. [Houston, 2016] }\end{array}$ & $\begin{array}{l}\text { De postgrado, tenemos } 137 \text { másteres, } \\
54 \text { doctorados, y } 3 \text { profesional programas } \\
\text { académicos. [P3] }\end{array}$ \\
\hline & $\begin{array}{l}\text { UH is located in Houston, Texas, the nation's } \\
\text { fourth-largest city and the energy capital of } \\
\text { the world. [Houston, 2016] }\end{array}$ & $\begin{array}{l}\text { UH está ubicación en Houston, Texas, en las } \\
\text { quanta más grande ciudad de la nación y } \\
\text { capital de la energía en el mundo. [P1] }\end{array}$ \\
\hline \multirow[t]{2}{*}{ Sustantivo con valor adjetivo } & $\begin{array}{l}\text { and our faculty routinely partner with busi- } \\
\text { nesses and government agencies through } \\
\text { research. [Houston, 2016] }\end{array}$ & $\begin{array}{l}\text { y nuestro profesorado rutinariamente socio } \\
\text { con empresas y agencias gobiernos a } \\
\text { través de investigación. [P5] }\end{array}$ \\
\hline & $\begin{array}{l}\text { Our undergraduates choose from } 120 \\
\text { majors and minors. [Houston, 2016] }\end{array}$ & $\begin{array}{l}\text { Para los programas grados, hay } 120 \\
\text { especializaciones y subespecializaciones } \\
\text { que podáis elegir. [P2] }\end{array}$ \\
\hline
\end{tabular}

En los ejemplos de anteposición de adjetivo podemos observar dos traducciones literales agramaticales como resultado del calco de las reglas sintácticas del inglés en la anteposición del adjetivo profesional y del sintagma adjetival más grande. Los ejemplos que presentan un sustantivo con valor adjetivo muestran dos intentos de adjetivación mediante el uso de los sustantivos gobiernos y grados pospuestos a los sustantivos agencias y programas. En términos generales, podemos afirmar que en español los adjetivos pueden aparecer tanto antepuestos como pospuestos al sustantivo que acompañan. En inglés, por el contrario, la posición del adjetivo es categórica, ya que este siempre debe preceder al sustantivo. Más espe-

${ }^{10}$ Se usan para los ejemplos las abreviaciones correspondientes a los tres textos de origen empleados y que se presentan en el Anexo C.

Estudios de Lingüística Aplicada, año 35, número 66, diciembre de 2017, pp. 205-232 doi: 10.22201/enallt.01852647p.2017.66.837 
cíficamente, en español identificamos dos grandes grupos de adjetivos: los calificativos y los relacionales. Según Demonte (1999), los adjetivos calificativos denotan cualidades o propiedades que se agregan al significado del sustantivo. Los adjetivos relacionales, por otro lado, aportan rasgos que permiten clasificar personas o cosas. Desde un punto de vista sintáctico, en función atributiva, es decir, unidos al sustantivo, los calificativos pueden aparecer tanto antepuestos como pospuestos a este (la agradable película o la película agradable), mientras que los relacionales siempre aparecen pospuestos (el plan académico). Otra de las diferencias entre el inglés y el español en el uso del adjetivo es que el inglés tiene una gran flexibilidad de adjetivación y permite que un sustantivo modifique a otro sustantivo.

A medida que avanza el curso, podemos observar cómo los estudiantes van progresivamente identificando los rasgos diferenciales entre el inglés y el español y respetando las reglas sintácticas del español. El siguiente ejemplo proviene de la última traducción asignada, llevada a cabo por los estudiantes durante la última semana de clase. Esta tarea consistía en la traducción de un folleto médico sobre la ansiedad.

\begin{tabular}{ll} 
CUADRO 5. Ejemplo de traducción que presenta posicionamiento del adjetivo y de adjetivación \\
\hline TEXTO DE ORIGEN & TEXTO META \\
\hline However, if you are often afraid or anxious for & Sin embargo, si a menudo tiene miedo o está \\
no reason and this is getting in the way of your & preocupada sin razón y eso también está in- \\
normal daily activities, you may have an anxiety & $\begin{array}{l}\text { terponiendo en sus actividades diarias norma- } \\
\text { disorder. [Anxiety, 2013] }\end{array}$ \\
& $\begin{array}{l}\text { les, entonces hay una posibilidad que tiene un } \\
\text { trastorno de ansiedad. [P3] }\end{array}$
\end{tabular}

En el Cuadro 5 se puede ver, por un lado, cómo el estudiante pospone adecuadamente los adjetivos diarias y normales que ocupan la posición antepuesta en el texto de origen. Por otro lado, en este fragmento también podemos observar cómo el estudiante realiza una transposición: el sustantivo anxiety se convierte en una frase preposicional con función adjetival de ansiedad.

Los siguientes ejemplos, mostrados en el Cuadro 6, provienen de una actividad de clase que tenía como objetivo trabajar la posición del adjetivo. Los estudiantes se dividieron en dos grupos y prepararon un borrador de la traducción. 
Después, los grupos intercambiaron los borradores y anotaron comentarios y sugerencias. Por último, cada grupo presentó una versión final de la traducción. ${ }^{11}$

En el Cuadro 6 podemos ver cómo los dos grupos posponen los adjetivos relacionales biológicas, amazónica, ecológicas y biológica. En el posicionamiento de los adjetivos calificativos, sin embargo, encontramos algunas pequeñas diferencias entre los dos grupos. El grupo A antepone el adjetivo calificativo espectaculares, mientras que el B lo pospone. Las dos soluciones respetan las reglas sintácticas del español, pero la anteposición denota más expresividad y subjetividad. Al final del texto, el grupo B decide anteponer el adjetivo calificativo inolvidable, probablemente para evitar la carga de dos adjetivos pospuestos, mientras que el grupo A opta por posponerlo y por usar el sintagma preposicional para los turistas en vez del adjetivo relacional turística. Estas elecciones sugieren que los estudiantes son capaces de distinguir las preferencias entre el inglés y el español en el posicionamiento del adjetivo.

CuADro 6. Ejemplos de traducción de los grupos A y B que presentan posicionamiento del adjetivo

\begin{tabular}{|c|c|c|}
\hline \multirow{2}{*}{ TEXTO DE ORIGEN } & \multicolumn{2}{|c|}{ TEXTO META } \\
\hline & Grupo A & Grupo B \\
\hline $\begin{array}{l}\text { The Noel Kempff Mercado National Park } \\
\text { is located in the north of Bolivia, in one } \\
\text { of the most diverse biological areas in } \\
\text { the world. This remote region, which } \\
\text { spreads from the Amazon rainforest to } \\
\text { spectacular waterfalls, contains hun- } \\
\text { dreds of rare and endangered species. } \\
\text { Located between wet and dry ecological } \\
\text { communities, the park is home to over } \\
130 \text { species of mammals and } 70 \text { species } \\
\text { of reptiles, including the black caiman. } \\
\text { A rich variety of trees bloom during the } \\
\text { whole year. The diversity of animals and } \\
\text { plants of the park make it an ideal place } \\
\text { for biological research and an unforget- } \\
\text { table attraction for tourists. [Adap. Lunn } \\
\& \text { Lunsford, 2003] }\end{array}$ & $\begin{array}{l}\text { El parque nacional Noel Kempff está } \\
\text { situado en el norte de Bolivia, en una } \\
\text { de las zonas biológicas más diversas } \\
\text { del mundo. Esta región remota, que } \\
\text { se extiende desde la selva Amazónica } \\
\text { hasta espectaculares cascadas, contie- } \\
\text { ne cientos de especies raras en peligro } \\
\text { de extinción. Situado entre las comu- } \\
\text { nidades ecológicas húmedas y secas, } \\
\text { el parque alberga más de } 130 \text { espe- } \\
\text { cies de mamíferos y } 70 \text { especies de } \\
\text { reptiles, incluyendo el caimán negro. } \\
\text { Una gran variedad de árboles florecen } \\
\text { durante todo el año. La diversidad de } \\
\text { animales y plantas del parque lo hacen } \\
\text { en un lugar ideal para la investigación } \\
\text { biológica y una atracción inolvidable } \\
\text { para los turistas. }\end{array}$ & $\begin{array}{l}\text { El parque nacional de Noel Kempff Mer- } \\
\text { cado se ubica al norte de Bolivia dentro } \\
\text { de una de las áreas biológicas más di- } \\
\text { versas del mundo. Esta región remota, } \\
\text { que se extiende desde la selva Ama- } \\
\text { zónica hasta las cascadas espectacu- } \\
\text { lares, y contiene cientos de especies } \\
\text { raras que están en vías de extinción. } \\
\text { Situado entre las comunidades ecoló- } \\
\text { gicas húmedas y secas, en el parque } \\
\text { habitan más de } 130 \text { especies de ma- } \\
\text { míferos y } 70 \text { especies de reptiles, el } \\
\text { cuál incluye el caimán negro (melano- } \\
\text { suchus niger). La selección rica y va- } \\
\text { riada de árboles florecen todo el año. } \\
\text { La diversidad de animales y plantas del } \\
\text { parque se hace la ubicación ideal para } \\
\text { las investigaciones biológicas y una in- } \\
\text { olvidable atracción turística. }\end{array}$ \\
\hline
\end{tabular}

11 Texto de origen adaptado de Lunn \& Lunsford (2003).

Estudios de Lingüística Aplicada, año 35, número 66, diciembre de 2017, pp. 205-232 doi: 10.22201/enallt.01852647p.2017.66.837 
Por último, se incluyen en (3) y (4) fragmentos de los proyectos finales del curso para ilustrar la capacidad de los estudiantes de analizar críticamente los factores extralingüísticos de las traducciones. Por limitaciones de espacio, solo se incluyen dos fragmentos:

(3) Porque sé que los traductores deben tener conocimientos sobre la cultura de la lengua de destino con el fin de determinar cuál es la estrategia de usar, mi punto de vista de la simplicidad de la traducción ha cambiado. De todos los factores que intervienen en la traducción, nada es más importante que la comprensión de la cultura y el dialecto de su audiencia meta. Las culturas influyen en las connotaciones positivas y negativas de determinadas palabras. Así que lo que puede considerarse aceptable en una cultura, puede ser permisible en otro. $\mathrm{Al}$ añadir esto a todos los demás factores de traducciones, las responsabilidades de un traductor comienzan a acumularse. (P5)

(4) Siempre pensé que las traducciones solamente eran un cambio de la lengua de origen a la lengua meta. No es tan fácil. Hay muchas cosas que necesita uno pensar antes de comenzar una traducción. En primer lugar, hay variables que considerar. Estos son los factores extralingüísticos. Estas son preguntas que necesitan ser hechas antes de comenzar una traducción. La función es el objetivo del texto. ¿Está tratando de enseñar, informar, o anunciar su punto? La audiencia es a los quién los lectores se dirigen el texto y sus características. Cada audiencia requiere diferentes cosas. Una persona de México no puede ser capaz de entender lo mismo que puede entender de una persona de España. Necesita satisfacer la audiencia en explicaciones para obtener el punto de texto a que usted quiera. (P2)

\section{CONCLUSIONES Y RECOMENDACIONES PEDAGÓGICAS}

Los resultados obtenidos en esta investigación ponen de manifiesto que la traducción, entendida en un sentido amplio, es una actividad a la que los estudiantes de segundas lenguas recurren naturalmente y con frecuencia en diferentes contextos. La mayoría de los estudiantes desempeñan tareas de interpretación en contextos variados como el trabajo, la universidad y el hogar, mientras que algunos de ellos emplean la traducción como actividad de clase y herramienta de estudio personal. Además de estar familiarizados con la traducción, sus acti- 
tudes ante dicha actividad son muy positivas; todos parecen reconocer su valor didáctico e instrumental. El análisis cuantitativo muestra que la incorporación de la traducción pedagógica mediante una metodología activa — que combine el trabajo individual y colaborativo y promueva la reflexión - tiene un impacto significativo en el desarrollo de habilidades lingüísticas en la segunda lengua. Particularmente, este impacto positivo se hace evidente en la habilidad de transferir información en la traducción inversa (L1 > L2) y en la habilidad de usar técnicas de traducción con mayores niveles de precisión. El análisis cualitativo sugiere que esta metodología permite que los estudiantes aborden la traducción como una actividad comunicativa definida por factores tanto lingüísticos como extralingüísticos y que desarrollen habilidades para identificar y respetar los rasgos diferenciales entre las dos lenguas, lo que a su vez les permite minimizar las traducciones literales agramaticales.

Teniendo en cuenta los resultados obtenidos, se proponen las siguientes recomendaciones pedagógicas para la integración de la actividad traductora en la enseñanza del español como segunda lengua:

1) Priorizar el proceso (la práctica de la traducción) sobre el producto (el texto traducido) mediante la puesta en práctica de una metodología activa y colaborativa.

2) Adoptar una evaluación formativa.

3) Promover el avance paulatino de actividades de reformulación o paráfrasis hacia actividades de traducción.

4) Introducir textos de géneros diversos.

5) Hacer evidentes los rasgos diferenciales entre el inglés y el español mediante el análisis contrastivo de textos de origen y sus traducciones.

6) Subrayar la importancia de los factores extralingüísticos que rodean los textos de origen y sus traducciones.

7) Fomentar el análisis crítico de las traducciones mediante comentarios acerca de la traducción. 


\section{LIMITACIONES DEL ESTUDIO}

Si bien este estudio preliminar presenta resultados interesantes, el número de participantes fue limitado y no incluyó un grupo de control. Para futuras investigaciones sería recomendable aumentar el número de participantes y añadir un grupo de control.

\section{REFERENCIAS}

CARreres, Ángeles (2006). Strange bedfellows: Translation and language teaching. The teaching of translation into L2 in modern languages degrees; uses and limitations. En Sixth symposium on translation, terminology and interpretation in Cuba and Canada. Ontario: Canadian Translators, Terminologists and Interpreters Council (CTTIC). Recuperado de http://www.cttic. org/ACTI/2006/papers/Carreres.pdf

Carreres, Ángeles, \& Noriega Sánchez, María (2011). Translation in language teaching: Insights from professional translator training. The Language Learning Journal, 39(3), 281-297. doi: 10.1080/09571736.2011.567356

Carreres, Ángeles, \& Noriega Sánchez, María (2012). Traducción pedagógica y pedagogía de la traducción: un diálogo necesario. En Actas XXIII Congreso Internacional de la Asele (pp. 253-261). Recuperado de http://cvc.cervantes.es/ensenanza/biblioteca_ele/asele/ pdf/23/23_0026.pdf

Colina, Sonia (2002). Second language acquisition, language teaching and translation studies. The Translator, 8(1), 1-24. doi:10.1080/13556509.2002.10799114

Colina, Sonia (2015). Fundamentals of translation. Cambridge: Cambridge University Press.

Cook, Guy (2010). Translation in language teaching: The argument for reassessment. Oxford: Oxford University Press.

Cummins, Jim (2008). Teaching for transfer: Challenging the two solitudes assumption in bilingual education. En Jim Cummins \& Nancy H. Hornberger (Eds.), Encyclopedia of language and education (pp. 65-77). Nueva York: Springer Science.

Demonte, Violeta (1999). El adjetivo: clases y usos. La posición del adjetivo en el sintagma nominal. En Ignacio Bosque, Violeta Demonte \& Real Academia Española (Eds.), Gramática descriptiva de la lengua española 1: sintaxis básica de las clases de palabras (pp. 129-213). Madrid: Espasa-Calpe.

Duff, Alan (1989). Translation. Oxford: Oxford University Press.

Estudios de Lingüística Aplicada, año 35, número 66, diciembre de 2017, pp. 205-232 doi: 10.22201/enallt.01852647p.2017.66.837 
González Davies, María (2002). Translation in foreign language learning: Sleeping with the enemy? APAC of News, 44, 64-74.

González Davies, María (2003). Secuencias: tareas para el aprendizaje interactivo de la traducción especializada. Barcelona: Octaedro.

González Davies, María (2004). Multiple voices in the translation classroom: Activities, tasks and projects. Ámsterdam: John Benjamins.

González Davies, María (2017). A collaborative pedagogy for translation. En Lawrence Venuti (Ed.), Teaching translation: Program, courses, pedagogies (pp. 71-78). Nueva York: Routledge.

Hurtado Albir, Amparo (2001). Traducción y traductología: introducción a la traductología. Madrid: Cátedra.

KiRAly, Donald C. (2000). A social constructivist approach to translator education: Empowerment from theory to practice. Manchester: St. Jerome.

LEONARDI, VANESSA (2010). The role of pedagogical translation in second language acquisition. From theory to practice. Berlín: Peter Lang.

Lunn, Patricia Vining, \& Lunsford, ERnest Jackson (2003). En otras palabras: perfeccionamiento del español por medio de la traducción. Washington: Georgetown University Press.

MAlmkJAer, KirSTEN (1998). Translation \& language teaching: Language teaching \& translation. Manchester: St. Jerome.

Sewell, Penelope (2004). Students buzz round the translation class like bees round the honey pot - why? En Kirsten Malmkjaer (Ed.), Translation in undergraduate degree programmes (pp. 151-162). Ámsterdam: John Benjamins.

VAldÉs, GuAdalupe (2003). Expanding definitions of giftedness: the case of young interpreters from immigrant communities. Mahwah: Lawrence Erlbaum.

Washbourne, Kelly (2009). Manual of Spanish-English translation. Boston: Prentice Hall.

Widdowson, Henry G. (2003). Defining issues in English language teaching. Oxford: Oxford University Press.

ZoJer, HeIdi (2009). The methodological potential of translation in second language acquisition: Re-evaluating translation as a teaching tool. En Arnd Witte, Theo Harden \& Alessandra Ramos de Oliveira Harden (Eds.), Translation in second language learning and teaching (pp. 31-52). Berna: Peter Lang.

Estudios de Lingüística Aplicada, año 35, número 66, diciembre de 2017, pp. 205-232 doi: 10.22201/enallt.01852647p.2017.66.837 


\section{AneXos}

Anexo A. Cuestionario sobre hábitos y actitudes con respecto a la traducción

\begin{tabular}{|c|c|c|c|c|}
\hline 1. How often do you translate aloud from English into Spanish? & Rarely & Monthly & Weekly & Daily \\
\hline \multicolumn{5}{|c|}{$\begin{array}{l}\text { 1.1. Please explain briefly in which contexts: } \\
\text { (e. g. at home, at work, in class, with friends, with family, with classmates, on my own, etc.) }\end{array}$} \\
\hline $\begin{array}{l}\text { 2. How often do you translate aloud translations from Spanish } \\
\text { into English? }\end{array}$ & Rarely & Monthly & Weekly & Daily \\
\hline \multicolumn{5}{|c|}{$\begin{array}{l}\text { 2.1. Please explain briefly in which contexts: } \\
\text { (e. g. at home, at work, in class, with friends, with family, with classmates, on my own, etc.) }\end{array}$} \\
\hline $\begin{array}{l}\text { 3. How often do you do written translations from English into } \\
\text { Spanish? }\end{array}$ & Rarely & Monthly & $\bigcirc^{\text {Weekly }}$ & Daily \\
\hline \multicolumn{5}{|c|}{$\begin{array}{l}\text { 3.1. Please explain briefly in which contexts: } \\
\text { (e. g. at home, at work, in class, with friends, with family, with classmates, on my own, etc.) }\end{array}$} \\
\hline $\begin{array}{l}\text { 4. How often do you do written translations from Spanish into } \\
\text { English? }\end{array}$ & Rarely & Monthly & Weekly & $\begin{array}{c}\text { Daily } \\
\bigcirc\end{array}$ \\
\hline \multicolumn{5}{|c|}{$\begin{array}{l}\text { 4.1. Please explain briefly in which contexts: } \\
\text { (e. g. at home, at work, in class, with friends, with family, with classmates, on my own, etc.) }\end{array}$} \\
\hline 5. Do you think translation is important? Please explain. & & & & \\
\hline
\end{tabular}

Anexo B. Texto de origen para la actividad pre-test/post- test de traducción inversa

\section{New iPad Air}

A new iPad Air appears to be on the way. According to some sources, Apple is likely to introduce an updated model of the Air during an event in mid-March, marking the line's first change in over a year. The Air 2 was released in October 2014, but Apple chose not to introduce an Air 3 this past fall, when it announced the iPad Pro and iPad Mini 4. There aren't many details on what to expect from the new iPad model, but the "9to$5 \mathrm{Mac}$ " site points to earlier rumors saying the new Air may get additional speakers and a flash for its rear camera. Most notably, the site says that Apple has been testing a version of the Air that works with the Apple Pencil. It's not entirely surprising that Apple would want to bring that feature from the Pro to the standard Air, but it would be surprising to see that happen after just a few months.

Estudios de Lingüística Aplicada, año 35, número 66, diciembre de 2017, pp. 205-232 doi: 10.22201/enallt.01852647p.2017.66.837 
Anexo C. Textos de origen usados durante el curso para los ejercicios de traducción

Texto de origen 1. University of Houston

Founded in 1927, the University of Houston is the leading public research university in the vibrant international city of Houston. Each year, we educate more than 40,750 students in more than 300 undergraduate and graduate academic programs, on campus and online. UH awards more than 8,000 degrees annually, with more than 230,000 alumni.

UH is located in Houston, Texas, the nation's fourth-largest city and the energy capital of the world. Our students regularly test their skills through internships with national and international companies based here, and our faculty routinely partner with businesses and government agencies through research.

Our undergraduates choose from 120 majors and minors. At the graduate level, we offer 139 master's, 54 doctoral, and three professional degree programs. You may study online through our Distance Education program, or take noncredit courses through Continuing Education. Many of our academic programs rank among the nation's best.

Our faculty and students conduct research through 25 research centers and in every academic department. UH research regularly breaks new ground and opens doors to new ways of understanding the world.

Texto de origen 2. Anxiety disorder

Anxiety can be a normal "alarm system" that alerts you to danger. However, if you are often afraid or anxious for no reason and this is getting in the way of your normal daily activities, you may have an anxiety disorder.

A Generalized Anxiety Disorder involves feeling tense and worried either frequently or constantly when there are no signs of trouble or any specific purpose for the worrying.

An anxiety disorder can be caused by a chemical imbalance in your body, an unconscious memory, or a side effect of a medicine or illness.

Symptoms of Anxiety:

- Fast heartbeat, sweating, or shortness of breath

- Trouble concentrating or having mind go "blank"

- Muscle tension or muscle aches
- Constant worry or obsession over small or large concerns

- Stomach and intestinal problems including diarrhea 
- Restlessness, irritability or feeling on edge

- Loss of sleep

- Shaking or feeling easily startled

How can I lessen my anxiety?

Psychotherapy and medication are used to treat anxiety, but simple lifestyle changes can also help.

- Get plenty of sleep.

- Avoid stimulants. Stimulants include caffeine, alcohol, drugs, over-the-counter diet pills, and certain cough and cold medicines.

- Control your worry. Pick a specific time to worry. Focus on what's actually happening, rather than what "might" happen. Then let go of the worry and go on with your day.
- Exercise everyday.

- Relax. Relax by practicing deep breathing, guided imagery, positive thinking, muscle relaxation or listening to relaxing music. There are handouts for learning to relax.

- Explore possible treatment plans with your health care provider. There may be behavioral therapies or medications available to lessen your anxiety.

Texto de origen 3. The Noel Kempff Mercado National Park

The Noel Kempff Mercado National Park is located in the north of Bolivia, in one of the most diverse biological areas in the world. This remote region, which spreads from the Amazon rainforest to spectacular waterfalls, contains hundreds of rare and endangered species. Located between wet and dry ecological communities, the park is home to over 130 species of mammals and 70 species of reptiles, including the black caiman. A rich variety of trees bloom during the whole year. The diversity of animals and plants of the park make it an ideal place for biological research and an unforgettable attraction for tourists. 


\section{Anexo D. Clasificación de las técnicas de traducción}

\begin{tabular}{|c|c|c|}
\hline TÉCNICA DE TRADUCCIÓN & DEFINIIIÓN & EJEMPLO \\
\hline Adaptación & $\begin{array}{l}\text { Se reemplaza un elemento cultural por otro propio } \\
\text { de la cultura receptora. }\end{array}$ & $\begin{array}{l}\text { TO: major } \\
\text { TM: especialización }\end{array}$ \\
\hline Amplificación & $\begin{array}{l}\text { Se introducen precisiones no formuladas en el } \\
\text { texto original (es probable que la cultura receptora } \\
\text { las necesite). }\end{array}$ & $\begin{array}{l}\text { TO: Apple is likely to introduce an upda- } \\
\text { ted model of the Air during an event in } \\
\text { mid-March. } \\
\text { TM: La compañía Apple a lo mejor pre- } \\
\text { sentara el modelo nuevo del Air durante } \\
\text { un evento en marzo. }\end{array}$ \\
\hline Elisión & $\begin{array}{l}\text { Se eliden elementos de información presentes en } \\
\text { el texto original (es probable que la cultura recep- } \\
\text { tora no necesite esa información). }\end{array}$ & $\begin{array}{l}\text { TO: La compañía Apple a lo mejor pre- } \\
\text { sentara el modelo nuevo del Air durante } \\
\text { un evento en marzo. } \\
\text { TM: Apple is likely to introduce an up- } \\
\text { dated model of the Air during an event } \\
\text { in mid-March. }\end{array}$ \\
\hline Calco & $\begin{array}{l}\text { Se traduce literalmente una palabra o sintagma } \\
\text { extranjero. }\end{array}$ & $\begin{array}{l}\text { TO: Apple Pencil } \\
\text { TM: Lapiz de Apple }\end{array}$ \\
\hline Compensación & $\begin{array}{l}\text { Se introduce en otro lugar del texto traducido un } \\
\text { elemento de información o efecto estilístico que } \\
\text { no se ha podido reflejar en el mismo lugar en que } \\
\text { aparece situado en el TO. }\end{array}$ & $\begin{array}{l}\text { TO: Selvas impresionantes, ríos cauda- } \\
\text { losos y reliquias históricas } \\
\text { TM: Spectacular rain forests, large tee- } \\
\text { ming rivers and vestiges of the past }\end{array}$ \\
\hline Creación discursiva & $\begin{array}{l}\text { Se establece una equivalencia totalmente imprevi- } \\
\text { sible fuera de contexto. }\end{array}$ & $\begin{array}{l}\text { TO: Rumble Fish } \\
\text { TM: La ley de la calle }\end{array}$ \\
\hline Descripción & $\begin{array}{l}\text { Se reemplaza un término o expresión por la des- } \\
\text { cripción de su forma y/o función. }\end{array}$ & $\begin{array}{l}\text { TO: Additional speakers and a flash for } \\
\text { its rear camera. } \\
\text { TM: Vocinas adicionales y una luz foto- } \\
\text { gráfica para la camera de atrás. }\end{array}$ \\
\hline Equivalente acuñado & $\begin{array}{l}\text { Se utiliza un término o expresión reconocido como } \\
\text { equivalente en la LM. }\end{array}$ & $\begin{array}{l}\text { TO: A new iPad Air appears on the way } \\
\text { TM: El Nuevo iPad Air parece que ya } \\
\text { viene en camino }\end{array}$ \\
\hline Generalización & Se utiliza un término más general o neutro. & $\begin{array}{l}\text { TO: Primavera } \\
\text { TM: Estación del año }\end{array}$ \\
\hline Particularización & Se utiliza un término más preciso o concreto. & $\begin{array}{l}\text { TO: Estación del año } \\
\text { TM: Primavera }\end{array}$ \\
\hline
\end{tabular}

Estudios de Lingüística Aplicada, año 35, número 66, diciembre de 2017, pp. 205-232 
(continuación)

\begin{tabular}{|c|c|c|}
\hline TÉCNICA DE TRADUCCIÓN & DEFINIIIÓN & EJEMPLO \\
\hline Modulación & $\begin{array}{l}\text { Se efectúa un cambio de punto de vista, de en- } \\
\text { foque o de categoría de pensamiento en relación } \\
\text { con la formulación del texto original. }\end{array}$ & $\begin{array}{l}\text { TO: The Air } 2 \text { was released in October } \\
2014 \\
\text { TM: El Air } 2 \text { salió en octubre del } 2014\end{array}$ \\
\hline Préstamo & $\begin{array}{l}\text { Se integra una palabra o expresión de otra lengua } \\
\text { tal cual. Puede ser puro (sin ningún cambio) o na- } \\
\text { turalizado (transliteración de la lengua extranjera). }\end{array}$ & $\begin{array}{l}\text { T0: The new iPad Air } \\
\text { TM: El nuevo iPad Air }\end{array}$ \\
\hline Traducción literal & $\begin{array}{l}\text { Se traduce palabra por palabra un sintagma } 0 \\
\text { expresión. }\end{array}$ & $\begin{array}{l}\text { TO: The Air } 2 \text { was released in October } \\
2014 \text {. } \\
\text { TM: El Air } 2 \text { fue lanzado en octubre del } \\
2014 \text {. }\end{array}$ \\
\hline Transposición & $\begin{array}{l}\text { Se cambia la categoría gramatical sin cambiar } \\
\text { el sentido. Puede ser obligatoria, exigida por la } \\
\text { lengua, o facultativa, resultado de una elección } \\
\text { estilística por parte del traductor. }\end{array}$ & $\begin{array}{l}\text { TO: Apple is likely to introduce an up- } \\
\text { dated model of the Air during an event } \\
\text { in mid-March, marking the line's first } \\
\text { change in over a year. } \\
\text { TM: Es muy posible que Apple introduzca } \\
\text { su nuevo modelo actualizado de Air du- } \\
\text { rante un evento en mediados de marzo. } \\
\text { Éste será el primer cambio de la línea } \\
\text { en un año. }\end{array}$ \\
\hline
\end{tabular}

Nota: LM, lengua meta; TM, texto meta T0, texto de origen.

Estudios de Lingüística Aplicada, año 35, número 66, diciembre de 2017, pp. 205-232 doi: 10.22201/enallt.01852647p.2017.66.837 